\title{
Administration of Intravenous Iron Formulations Induces Complement Activation in-vivo
}

\author{
Bernardo Faria ${ }^{1,2,3 \dagger}$, Mariana Gaya da Costa ${ }^{1 \dagger}$, Felix Poppelaars ${ }^{1}$, Casper F. M. Franssen ${ }^{1}$, \\ Manuel Pestana ${ }^{3}$, Stefan P. Berger ${ }^{1}$, Mohamed R. Daha ${ }^{1,4}$, Carlo A. J. M. Gaillard ${ }^{5}$ and \\ Marc A. Seelen ${ }^{1 *}$ \\ ${ }^{1}$ Division of Nephrology, Department of Internal Medicine, University Medical Center Groningen, University of Groningen, \\ Groningen, Netherlands, ${ }^{2}$ Department of Nephrology, Hospital de Braga, Braga, Portugal, ${ }^{3}$ Nephrology and Infectious \\ Disease R\&D Group, INEB, I3S, University of Porto, Porto, Portugal, ${ }^{4}$ Department of Nephrology, Leiden University Medical \\ Center, University of Leiden, Leiden, Netherlands, ${ }^{5}$ Division of Internal Medicine and Dermatology, University Medical Center \\ Utrecht, University of Utrecht, Utrecht, Netherlands
}

OPEN ACCESS

Edited by:

Robert Braidwood Sim University of Oxford, United Kingdom

Reviewed by: Andrea Doni, Humanitas Research Hospital, Italy Lubna Kouser,

Imperial College London, United Kingdom

*Correspondence:

Marc A. Seelen m.seelen@umcg.nl

these authors have contributed equally to this work

Specialty section:

This article was submitted to Molecular Innate Immunity, a section of the journal

Frontiers in Immunology

Received: 24 April 2019 Accepted: 25 July 2019

Published: 21 August 2019

Citation:

Faria B, Gaya da Costa M

Poppelaars F, Franssen CFM, Pestana M, Berger SP, Daha MR, Gaillard CAJM and Seelen MA (2019) Administration of Intravenous Iron Formulations Induces Complement Activation in-vivo.

Front. Immunol. 10:1885. doi: 10.3389/fimmu.2019.01885
Background: Intravenous (IV) iron is widely used to treat anemia in chronic kidney disease patients. Previously, iron formulations were shown to induce immune activation in-vitro. The current study aimed to investigate the effect of IV iron on complement activation in-vivo, and whether this subsequently induces inflammation and/or oxidative stress.

Methods: Two distinct patient groups were included: 51 non-dialysis and 32 dialysis patients. The non-dialysis group received iron sucrose or ferric carboxymaltose, based on physicians' choice. Plasma samples were collected prior to and $1 \mathrm{~h}$ after completion of IV iron infusion. The dialysis group received iron sucrose exclusively. Plasma samples were collected at the start and end of two consecutive hemodialysis sessions, one with and one without IV iron. Finally, plasma levels of MBL, C1q, properdin, factor D, sC5b-9, MPO, PTX3 were assessed by ELISA.

Results: In the non-dialysis group, sC5b-9 levels significantly increased after IV iron by $32 \%$, while levels of factor $\mathrm{D}$ and $\mathrm{MBL}$ significantly dropped. Subgroup analysis demonstrated that iron sucrose induced complement activation whereas ferric carboxymaltose did not. In the dialysis group, levels of sC5b-9 significantly increased by $46 \%$ during the dialysis session with IV iron, while factor $D$ levels significantly fell. Furthermore, the relative decrease in factor $\mathrm{D}$ by IV iron correlated significantly with the relative increase in sC5b-9 by IV iron. MPO levels rose significantly during the dialysis session with IV iron, but not in the session without iron. Moreover, the relative increase in MPO and sC5b-9 by IV iron correlated significantly. PTX3 levels were not affected by IV iron.

Conclusions: Iron sucrose but not ferric carboxymaltose, results in complement activation possibly via the lectin and alternative pathway partially mediating oxidative stress but not inflammation.

Keywords: kidney, complement, hemodialysis, iron, anemia and kidney disease 


\section{INTRODUCTION}

Intravenous (IV) iron drugs are a mainstay in the management of anemia (1). A growing number of chronic kidney disease (CKD) patients receive IV iron $(1,2)$. In the past, safety concerns existed, since IV iron has been linked to iron overload, increased oxidative stress, cardiovascular risk, infection risk, and hypersensitivity reactions $(1,3)$. Recently, a multicenter open-label clinical trial with over 2,000 hemodialysis (HD) patients demonstrated that a high-dose IV iron sucrose regimen administered pro-actively resulted in lower cardiovascular events (29 vs. $32 \%$ ) when compared to a low-dose IV iron sucrose regimen, while no difference in infection and mortality was seen (4). However, some questions persist about the long-term safety of IV iron, the comparison of different iron formulations and the clinical outcome in non-dialysis patients. Therefore, a better understanding of the IV iron (side) effects remains a relevant subject of research.

Previously, hypersensitivity reactions induced by IV iron have been proposed to arise from complement activationrelated pseudo-allergy (CARPA) (5). Complement activation can be initiated via three different pathways: the classical pathway (CP), the lectin pathway (LP), and the alternative pathway (AP). All the pathways converge at the level of C3 and ultimately results in generation of C5b-9, the membrane attack complex (MAC) (6). Hypersensitivity reactions are extremely rare, making it difficult to establish a cohort large enough to investigate the CARPA hypothesis (7). Nevertheless, the concept of complement activation by IV iron warrants further investigation. A few studies have previously shown the ability of IV iron formulations to activate complement in-vitro $(8,9)$. For instance, low-molecular weight iron dextran, ferric gluconate, ferric carboxymaltose, and iron sucrose were all shown to activate complement. However, the paradoxical results found in these studies, emphasize the need for further research.

The current study aimed to investigate the effect of IV iron on complement activation in-vivo. In addition, we explored if a potential IV iron induced complement activation could lead to oxidative stress and inflammation. Therefore, complement activation was assessed through the measurement of sC5b-9 levels and components of different pathways, namely $\mathrm{C} 1 \mathrm{q}$ from the CP, MBL from the LP and factor D and properdin from the AP. Also, inflammation and oxidative stress were studied through the measurement of myeloperoxidase (MPO) and pentraxin-3 (PTX-3). All these measurements were performed in distinct groups receiving IV iron treatment.

\footnotetext{
Abbreviations: AP, Alternative pathway; CARPA, Complement activation related pseudoallergy; CKD, Chronic kidney disease; ND-CKD, Non-dialysis chronic kidney disease; CP, Classical pathway; CRP, C reactive protein; EDTA, Ethylenediaminetetraacetic acid; HD, Hemodialysis; IL-6, Interleukin-6; IV, Intravenous; LP, Lectin pathway; MAC, Membrane attack complex; MBL, Mannose-binding lectin; MPO, Myeloperoxidase; PTX3, Pentraxin-3; sC5b-9, soluble membrane attack complex; SEM, Standard error of the mean; TNF- $\alpha$, Tumor necrosis factor alpha.
}

\section{MATERIALS AND METHODS}

\section{Study Design and Population}

Patients were recruited from the day clinic and the dialysis unit of Hospital de Braga, Braga, Portugal. The first group included nondialysis patients (CKD and non-CKD), receiving iron sucrose (Venofer ${ }^{\circledR}$, Vifor Pharma) or ferric carboxymaltose (Ferinject ${ }^{\circledR}$, Vifor Pharma) and the second group consisted of dialysis patients receiving only iron sucrose. Adult patients ( $\geq 18$ years) receiving IV iron and who gave informed consent were eligible for the study. Exclusion criteria were administration of iron in the previous 3 months for the first group whereas signs of active inflammation was exclusion criteria for both groups.

In the non-dialysis group, the drug type and the dose of IV iron was based on the decision of the treating physician according to local clinical guidelines and protocols. Iron was infused with a slow infusion rate and complete drug administration occurred in $30 \mathrm{~min}$ for $100 \mathrm{mg}$ of iron sucrose and $500 \mathrm{mg}$ of ferric carboxymaltose, whereas higher doses of both drugs were infused in $1 \mathrm{~h}$. Plasma EDTA samples were collected prior to and $1 \mathrm{~h}$ after completion of the iron infusion. During this period, patients were observed for allergic symptoms and signs, including itching, arthralgias, hypotension, tachycardia, respiratory symptoms, thoracic pain, edema, and/or rash.

In the dialysis group, patients received onlinehemodiafiltration three times per week for $4 \mathrm{~h}$ using high flux polysulfone dialyzers (Fx80, Helixone, Fresenius Medical Care, St. Wendel, Germany). Two patients were dialyzed with cellulose triacetate filters (CT190G, Baxter, McGaw Park, IL, USA). Patients received iron sucrose starting $3 \mathrm{~h}$ after the start of the dialysis session through a slow IV injection over 2 min via the venous limb of the circuit. The treating physician decided the dose of the IV iron formulation. Samples were collected during two consecutive sessions, one with IV iron administration and one without. During each dialysis session, plasma EDTA samples were collected prior to dialysis (pre-dialysis) and after $4 \mathrm{~h}$, at the end of the session (post-dialysis).

All blood samples were centrifuged within $30 \mathrm{~min}$ of collection at $3500 \mathrm{rpm}$ for $15 \mathrm{~min}$. Next, the samples were stored in aliquots at $-80^{\circ} \mathrm{C}$ until the measurement of the different laboratory parameters. Prior to the assay, samples were thawed and re-centrifuged.

\section{Laboratory Procedures}

Levels of sC5b-9 levels, C1q, MBL, factor D, and properdin were quantified in plasma EDTA samples by in-house sandwich ELISA as previously described $(8,10)$. In brief, plates were coated overnight at $4{ }^{\circ} \mathrm{C}$ with the respective antibody in coating buffer (100 mM Na $2 \mathrm{CO}_{3} / \mathrm{NaHCO}_{3}, \mathrm{pH}$ 9.6). After coating, plates were blocked using 1\% BSA/PBS. Next, plasma samples were diluted into the appropriate buffer solution and incubated in the coated wells. The protein of choice was then detected using the DIG- or biotin-conjugated coating antibody followed by detection with streptavidin-poly-horseradish peroxidase or HRP-conjugated sheep anti-Dig Antibodies (Fab fragments, Roche, Mannheim, Germany). After each step the plate was washed in PBS Tween$20(0.05 \%)$ and all incubation steps were done for $1 \mathrm{~h}$ at 
$37^{\circ} \mathrm{C}$. For visualization $3,3^{\prime}, 5,5^{\prime \prime}$ - Tetramethylbenzidine (TMB) or 2,2'-azino-bis(3-ethylbenzothiazoline-6-sulphonic acid) (ABTS) was added and the optical density was measured at 450 or $415 \mathrm{~nm}$, respectively. For the $\mathrm{C} 1 \mathrm{q}$ and properdin ELISA, in house antibodies were used. For the other ELISAs anti-MBL $3 \mathrm{e} 7$ antibody (Hycult, Uden, The Netherlands), anti-factor D 18241 antibody (R\&D systems, Wiesbaden-Norderstedt, Germany) or anti-C5b-9 AE11 antibody (DAKO, Copenhagen, Denmark) was used. The capture antibody for the sC5b-9 ELISA was different from the coating antibody and instead a goat antihuman C5 antibody was used (Sigma-Aldrich, St. Louis, MO, USA). MPO and PTX3 were measured using commercial ELISA kits according to the manufacturers' instructions (Hycult Biotech, Uden, The Netherlands).

\section{Statistics}

Statistical analysis was performed using IBM SPSS 23.0 (IBM Corporation, Chicago, IL, USA). Laboratory measurements are shown as mean \pm standard error of the mean (SEM). Comparisons between samples were made by paired sample $t$-test. Correlations were assessed using Pearson correlation coefficient (r). $P$-values $<0.05$ were considered to be statistically significant. The ratios used for the analyses were calculated per patient by dividing the pre-dialysis level by the post-dialysis level in both sessions. Subsequently the relative increase was calculated dividing the ratio of the session with iron, by the session without.

\section{Ethics}

This study was performed in accordance to the Declaration of Helsinki and was approved by the Medical Ethical Committee from Hospital Braga. All participants signed informed written consent.

\section{RESULTS}

\section{Intravenous Infusion of Iron Sucrose Leads to Complement Activation in Non-dialysis Patients}

The effect of IV iron on the complement system was first assessed in the non-dialysis group. This consisted of 51 patients with a median age of 64 years of which $63 \%$ were female (Table 1). Fifteen had non-dialysis CKD (ND-CKD) and 36 were non-CKD patients with anemia due to gastrointestinal or gynecological origin. Seventeen patients received ferric carboxymaltose at doses of $500 \mathrm{mg}(n=3)$ or $1,000 \mathrm{mg}(n=14)$, while 34 received iron sucrose at doses of $100 \mathrm{mg}(n=13), 200 \mathrm{mg}(n=20)$ or $300 \mathrm{mg}$ $(n=1)$. No hypersensitivity reactions were observed.

Overall, IV iron administration resulted in significant higher levels of sC5b-9 compared to baseline ( $P=0.007$; Figure 1A). The mean baseline values were $65.9 \pm 17.1 \mathrm{ng} / \mathrm{mL}$ and rose to $87.3 \pm 11.3 \mathrm{ng} / \mathrm{mL}$ after infusion, representing an average increase of $32 \%$ in sC5b-9 levels by IV iron. Nevertheless, the magnitude of the changes in levels of sC5b-9 by iron infusion varied, with $84 \%$ of patients showing complement activation by IV iron (Figure 1B). Next, we performed subgroup analyses of $\mathrm{ND}-\mathrm{CKD}$ vs. non-CKD. In the ND-CKD group, levels of sC5b9 were significantly increased by iron treatment $(P=0.007$,
TABLE 1 | Baseline characteristics of the non-dialysis and dialysis patients.

\begin{tabular}{|c|c|c|}
\hline & $\begin{array}{c}\text { Non-dialysis } \\
(n=51)\end{array}$ & $\begin{array}{l}\text { Dialysis } \\
(n=32)\end{array}$ \\
\hline Age, years & $64.3 \pm 19.8$ & $69.4 \pm 15.7$ \\
\hline Female gender, $n$ (\%) & $32(63)$ & $9(28)$ \\
\hline Weight, kg & $69.3 \pm 13.6$ & $71.7 \pm 10.9$ \\
\hline BMI, $\mathrm{kg} / \mathrm{m}^{2}$ & $28.5 \pm 4.9$ & $26.5 \pm 4.2$ \\
\hline Diabetes, $n$ (\%) & $13(25)$ & $17(53)$ \\
\hline \multicolumn{3}{|l|}{ Anemia Etiology, $n(\%)$} \\
\hline Gastro-intestinal & $27(53)$ & 0 \\
\hline Gynecological & $9(18)$ & 0 \\
\hline Kidney Disease & $15(29)$ & $32(100)$ \\
\hline Mixed $^{*}$ & $8(16)$ & 4 (12) \\
\hline \multicolumn{3}{|l|}{ Stages of CKD (KDIGO), $n$ (\%) } \\
\hline III & $6(40)$ & 0 \\
\hline IV & $6(40)$ & 0 \\
\hline V & $3(20)$ & $32(100)$ \\
\hline Hemoglobin, g/dL & $9.7 \pm 2.1$ & $11.4 \pm 1.2$ \\
\hline Serum ferritin concentration, $\mathrm{ng} / \mathrm{mL}$ & $48.1 \pm 87.4$ & $357 \pm 188$ \\
\hline Transferrin saturation, \% & $10.8 \pm 8.3$ & $30.9 \pm 19.4$ \\
\hline \multicolumn{3}{|l|}{ Type of IV Iron, $\boldsymbol{n}(\%)$} \\
\hline Iron Sucrose & $34(67)$ & $32(100)$ \\
\hline $20 \mathrm{mg}$ & 0 & $4(12)$ \\
\hline $50 \mathrm{mg}$ & 0 & $15(47)$ \\
\hline $100 \mathrm{mg}$ & $13(25)$ & $13(41)$ \\
\hline $200 \mathrm{mg}$ & $20(39)$ & 0 \\
\hline $300 \mathrm{mg}$ & $1(2)$ & 0 \\
\hline Ferric Carboximaltose & $17(33)$ & 0 \\
\hline $500 \mathrm{mg}$ & $3(6)$ & \\
\hline $1,000 \mathrm{mg}$ & $14(27)$ & \\
\hline \multicolumn{3}{|l|}{ Other anemia treatments, $n(\%)$} \\
\hline Oral Iron supplementation & $18(35)$ & 0 \\
\hline Erythropoiesis stimulating agents & $6(12)$ & $30(94)$ \\
\hline
\end{tabular}

BMI, body mass index; CKD, Chronic Kidney Disease; KDIGO, Kidney Disease Improving Global Outcomes; IV, intravenous.

All patients were Caucasians and data is expressed as means \pm standard error of the mean or absolute numbers (percentage).

*nvolving more than one anemia etiology.

Figure 1C), whereas in the non-CKD there was a trend for higher levels of sC5b-9 by IV iron $(P=0.05)$. Furthermore, infusion of iron sucrose was compared to ferric carboxymaltose. IV infusion with iron sucrose resulted in a significant rise in sC5b-9 levels $(P<0.001$; Figure 1D). Baseline levels of sC5b9 were $53.6 \pm 6.6 \mathrm{ng} / \mathrm{mL}$ and after IV iron increased to 89.7 $\pm 7.1 \mathrm{ng} / \mathrm{mL}$, showing an increase of $67 \%$. In contrast, sC5b-9 levels were not significantly altered by iron infusion with ferric carboxymaltose $(P=0.76$; Figure 1D). Of note, baseline levels were not statistically different between patients receiving iron sucrose compared to ferric carboxymaltose.

To explore the effect of IV iron on complement components, $\mathrm{C} 1 \mathrm{q}$, properdin, factor $\mathrm{D}$, and $\mathrm{MBL}$ were measured. Iron infusion was associated with a significant fall in the levels of factor D $(P=0.007$; Figure 2A) and MBL compared to baseline $(P=$ 0.006; Figure 2B), whereas properdin and C1q levels were not significantly altered after IV iron administration (Table 2). 

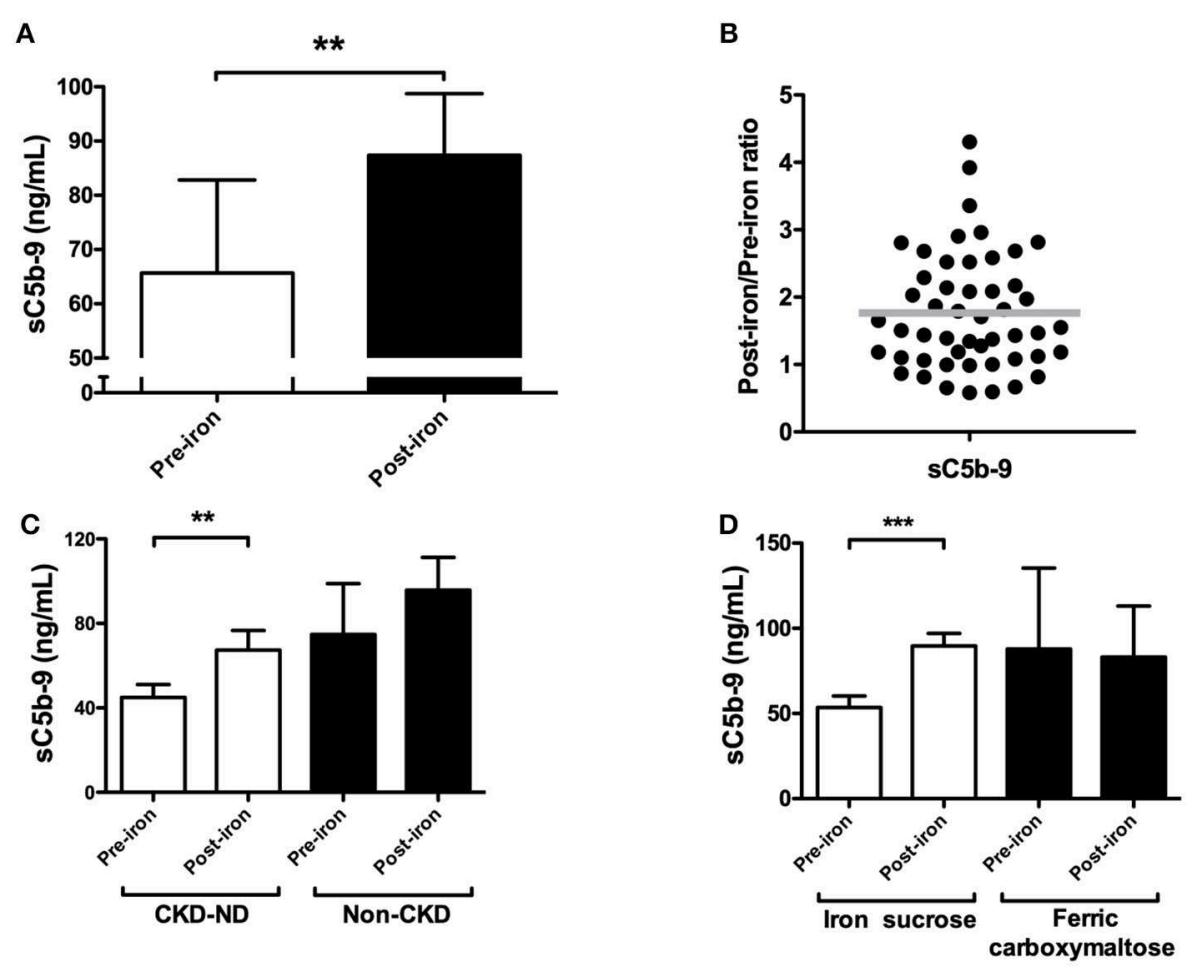

FIGURE 1 | The effect of IV iron on complement activation, as assessed by soluble C5b-9 in non-dialysis patients. (A) The plasma levels of soluble C5b-9 (sC5b-9) were determined in 51 patients prior to and $1 \mathrm{~h}$ after completion of iron infusion. (B) The ratio of sC5b-9 was calculated per patient by dividing the pre-iron level by the post-iron level. Horizontal lines indicate the mean. A post/pre-ratio higher than 1, indicates an increase in concentration by iron. Subgroup analysis of plasma sC5b-9 levels in (C) chronic kidney disease (CKD) patients $(n=15)$ vs. non-CKD patients $(n=36)$. (D) In patients receiving ferric carboxymaltose $(n=17)$ vs. iron sucrose $(n=34)$. Data are presented as mean and SEM (A,C,D). Paired sample t-test was used to compare values before and after iron infusion. P-values $<0.05$ were considered to be statistically significant $\left({ }^{\star *} P<0.01,{ }^{\star * *} P<0.001\right)$.
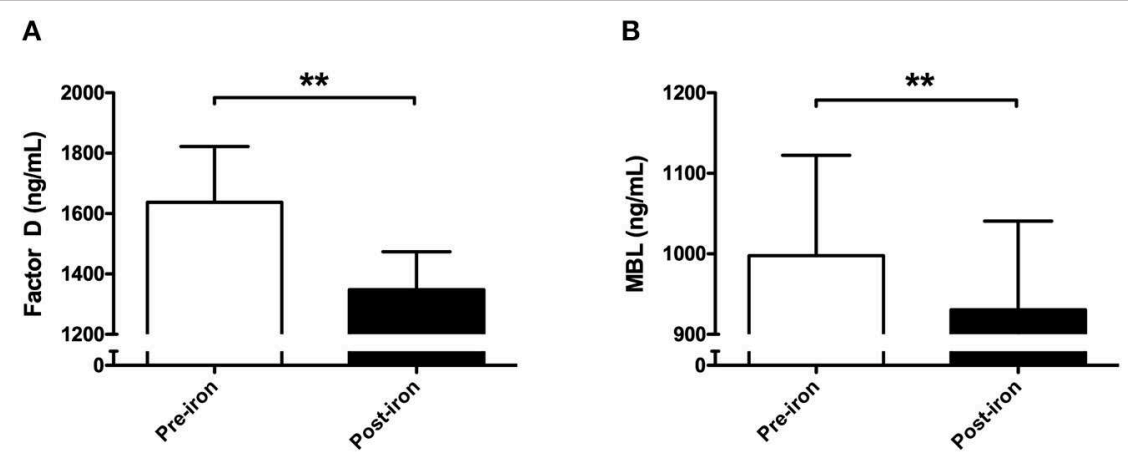

FIGURE 2 | Consumption of mannose-binding lectin and Factor D following intravenous iron in non-dialysis patients. In 51 non-dialysis patients plasma levels of (A) factor D and (B) mannose-binding lectin (MBL) were determined prior to and $1 \mathrm{~h}$ after completion of iron infusion. Data are presented as mean and SEM. Paired sample $t$-test was used to compare values before and after iron infusion. P-values $<0.05$ were considered to be statistically significant $\left({ }^{\star \star} P<0.01\right)$.

\section{Iron Infusion Has No Effect on Oxidative Stress or Inflammation in Non-dialysis Patients}

Subsequently, we determined the effect of IV iron on oxidative stress and inflammation by measuring levels of MPO and PTX3, respectively. Iron infusion resulted in a $33 \%$ increase of MPO levels, yet this rise was not statistically significant $(P=$
0.17; Figure 3A). PTX3 levels were unaffected by iron infusion, showing similar levels before and after iron infusion $(P=0.68$; Figure 3B). In subgroup analysis, infusion of iron sucrose led to a non-significantly increase of MPO levels by $41 \%$ ( $P=$ 0.18), while PTX3 levels were not affected. The use of ferric carboxymaltose did not significantly affect MPO or PTX3 levels (data now shown). 


\section{Intravenous Infusion of Iron Sucrose Leads to Complement Activation in Hemodialysis Patients}

Next, we analyzed the effect of iron sucrose on complement activation in 32 dialysis patients, at doses of $100 \mathrm{mg}(n=$

TABLE 2 | The effect of intravenous iron on C1q and properin in non-dialysis patients and the correlation with soluble C5b-9.

\begin{tabular}{lrrrrrrr}
\hline & \multicolumn{1}{c}{} & & & \multicolumn{2}{c}{ sC5b-9 } \\
\cline { 2 - 3 } & Pre-iron & Post-iron & $\boldsymbol{P}^{*}$ & & $\boldsymbol{R}$ & $\boldsymbol{P}^{\#}$ \\
\hline $\mathbf{C 1 q}(\mu \mathrm{g} / \mathrm{mL})$ & $69.4 \pm 4.5$ & $64.9 \pm 4.4$ & 0.20 & & 0.03 & 0.8 \\
Properdin $(\mu \mathrm{g} / \mathrm{mL})$ & $10.1 \pm 0.8$ & $8.8 \pm 0.5$ & 0.06 & -0.14 & 0.3 \\
\hline
\end{tabular}

Values are expressed as mean \pm standard error of the mean. $P^{\star}$ indicates $P$-values for difference between samples pre and post-iron tested by paired t-test. $R$ indicates Pearson correlation coefficient between Post-iron/Pre-iron ratio between the complement component and sC5b-9, and the corresponding P-value is shown by $P^{\#}$.
$13), 50 \mathrm{mg}(n=15)$, and $20 \mathrm{mg}(n=4)$. The mean age was 69 years and $72 \%$ were male (Table 1). During the study, no hypersensitivity reactions were observed in the dialysis patients. In the dialysis session without IV iron, sC5b-9 levels were not significantly increased after dialysis ( $P=0.27$; Figure 4A). However, IV iron during dialysis resulted in a significant rise of levels of sC5b-9 compared to baseline $(P=0.001)$ as well as compared to the end of the dialysis session without iron $(P=$ 0.002 ; Figure 4A). IV iron resulted in an increase of $46 \%$ in sC5b9 levels, compared to a $14 \%$ increase in the dialysis without IV iron. Of note, the magnitude of the change in sC5b-9 levels by iron infusion varied, with $60 \%$ of the dialysis patients showing complement activation by IV iron (Figure 4B).

Once again, levels of $\mathrm{C} 1 \mathrm{q}$, properdin, factor $\mathrm{D}$, and $\mathrm{MBL}$ were assessed to study the effect of IV iron on complement components (Table 3). Factor D levels did not significantly change during standard hemodialysis $(P=0.39$, Figure $5 \mathbf{A})$, but were significantly reduced after the dialysis session with IV iron infusion $(P=0.002$; Figure 5A). Moreover, the relative decrease
A

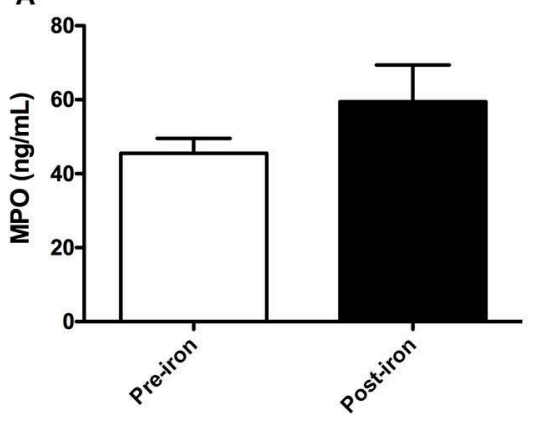

B

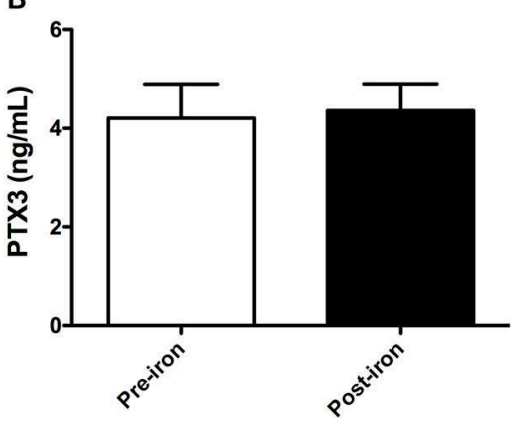

FIGURE 3 | Pentraxin-3 and myeloperoxidase are not altered by intravenous iron in non-dialysis patients. In 51 non-dialysis patients, plasma levels of (A) myloperoxidase (MPO) and (B) pentraxin-3 (PTX3) were determined prior to and $1 \mathrm{~h}$ after completion of iron infusion. Data are presented as mean and SEM. Paired sample t-test was used to compare values before and after iron infusion. $P$-values $<0.05$ were considered to be statistically significant.
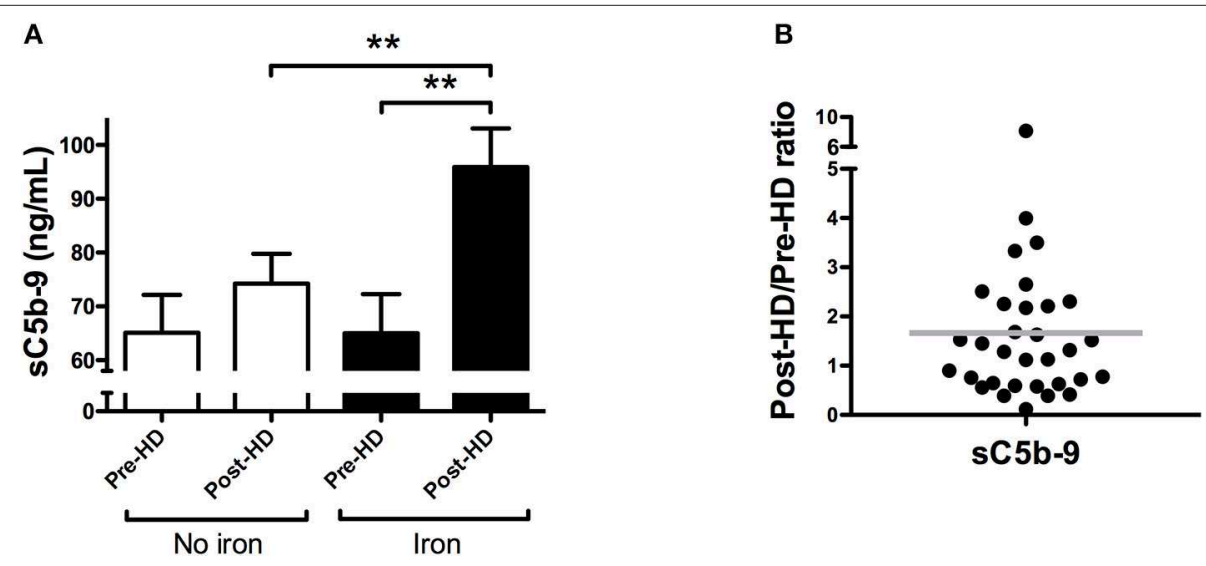

FIGURE 4 | Iron sucrose results in complement activation in dialysis patients. (A) Plasma levels of soluble C5b-9 (sC5b-9) were determined in 32 hemodialysis patients during two consecutive dialysis sessions, one with intravenous iron (Iron), and one without (No iron). During each dialysis session, sC5b-9 levels were determined at baseline and after $4 \mathrm{~h}$. Data are presented as mean and SEM. (B) The relative change in sC5b-9 was calculated per patient by dividing the pre-dialysis level by the post-dialysis level in both sessions and subsequently diving the session with iron, by the session without. Horizontal lines indicate the mean. A post/pre-ratio higher than one, indicates an increase in concentration of sC5b-9 by iron sucrose. The paired sample $t$-test was used to compare values before and after iron infusion. $P$-values $<0.05$ were considered to be statistically significant ( ${ }^{\star \star} P<0.01$ ). 
TABLE 3 | The effect of intravenous iron on complement levels in dialysis patients and the correlation with soluble C5b-9.

\begin{tabular}{|c|c|c|c|c|c|c|c|c|}
\hline & \multicolumn{3}{|c|}{ No iron } & \multicolumn{3}{|c|}{ Iron } & \multicolumn{2}{|c|}{ sC5b-9 } \\
\hline & Pre-HD & Post-HD & $P^{*}$ & Pre-HD & Post-HD & $\boldsymbol{P}^{\%}$ & $\boldsymbol{R}$ & $\boldsymbol{P}$ \\
\hline C1q $(\mu \mathrm{g} / \mathrm{mL})$ & $39.7 \pm 9.8$ & $43.2 \pm 10.0$ & 0.6 & $42.06 \pm 10.3$ & $40.8 \pm 9.3$ & 0.8 & -0.13 & 0.4 \\
\hline MBL(ng/mL) & $862 \pm 145$ & $1,087 \pm 229$ & 0.1 & $1,660 \pm 688$ & $1,518 \pm 570$ & 0.8 & 0.03 & 0.9 \\
\hline Properdin $(\mu \mathrm{g} / \mathrm{mL})$ & $9.1 \pm 1.2$ & $9.6 \pm 6.3$ & 0.8 & $11.4 \pm 1.4$ & $12.0 \pm 1.8$ & 0.6 & -0.30 & 0.4 \\
\hline
\end{tabular}

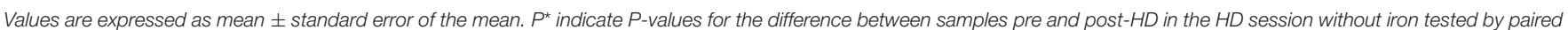

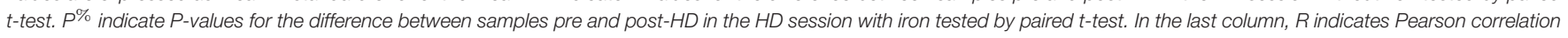
coefficient between Post-HD/Pre-HD ratio between the sessions of the complement component compared with the Post-HD/Pre-HD ratio between the sessions of sC5b-9.

of factor D by IV iron correlated with the relative increase of sC5b-9 by IV iron ( $r=0.49, P=0.004$; Figure 5B), suggesting that consumption of factor $\mathrm{D}$ is linked to complement activation. Administration of IV iron during dialysis did not significantly affect MBL, C1q or properdin levels (Table 3).

\section{Intravenous Iron Sucrose Leads to Higher MPO Levels in Hemodialysis Patients, and Is Correlated With Complement Activation}

The dialysis session without IV iron infusion showed no significant changes in MPO levels ( $P=0.36$; Figure 6A), while, the dialysis session with IV iron resulted in a significant rise in MPO levels $(P=0.02$; Figure 6A). Levels of MPO showed an increase of $47 \%$ compared to baseline. Moreover, the relative increase of MPO levels by IV iron correlated significantly with the relative increase in C5b-9 levels by IV iron $(r=0.42, P=0.02$; Figure 6B). Levels of PTX3 were already significantly increased by dialysis itself $(P=0.002$; Figure $6 \mathrm{C})$ and administration of IV iron did not result in a further increase.

\section{DISCUSSION}

The current study demonstrates, for the first time, that administration of iron sucrose, but not ferric carboxymaltose, results in complement activation in-vivo. Iron sucrose induced complement activation in both non-dialysis and dialysis patients. However, while in the majority of patients iron sucrose led to complement activation, the magnitude of the response to IV iron varied considerably among the patients. Complement activation induced by iron sucrose is possibly mediated via the AP, since factor D consumption by IV iron correlated with increased levels of sC5b-9. However, involvement of the LP cannot be excluded since iron infusion in the non-dialysis patients resulted in lower MBL levels. Finally, iron sucrose significantly increased MPO levels during dialysis and the relative increase of MPO correlated with complement activation. This finding suggests that complement activation induced by IV-iron is linked to neutrophil activation resulting in oxidative stress.

Previously, our group tested the in-vitro ability of different iron formulations to activate the complement system (8). Based on the in-vitro studies, complement activation by iron sucrose was thought to occur via LP activation. The current findings implicate AP and possibly LP activation by iron sucrose. We found a significant reduction of factor D by IV iron and also a trend for lower properdin in the non-dialysis group, implying AP involvement. The significant decrease in MBL levels in the non-dialysis group could suggest a role for LP activation by iron sucrose. Recent studies indicate that activation of the AP can be mediated via the LP, more specifically initiation of the MBLMASP complex can result in the conversion of pro-factor D in factor D by MASP-3 (11). The mechanisms behind complement activation induced by iron drugs are still unclear. A crucial difference between the iron formulations are the carbohydrate ligands which impacts the immunoreactivity as well as the stability of the molecule and the release of iron (12). In our study, iron sucrose was shown to be a complement activator while ferric carboxymaltose was not. In conformity, Fell et al. demonstrated that iron sucrose was the only formulation to induce monocyte activation (13). We propose that low stability and high labile iron release are major determinants for complement activation by IV iron. Therefore, we would expect that of the iron drugs that were not tested yet, low-molecular weight iron dextran and ferric gluconate lead to complement activation, but not ferumoxytol and iron isomaltoside.

The use of IV iron led to complement activation in the majority of the patients, however, the individual response to iron varied per patient. At present, it is not clear which determinants affect the degree of complement activation. Based on our findings, the role of the underlying cause of anemia seems limited, since complement activation was seen in non$\mathrm{CKD}, \mathrm{ND}-\mathrm{CKD}$, and dialysis patients. Furthermore, the rate of infusion has been previously shown to be crucial for both, hypersensitivity reactions and complement activation (14-16). However, considering current infusion protocols and newer iron formulations, the administration rate cannot explain the complement activation by IV iron seen in this study. In addition, the dose of iron could also affect the extent of complement activation. We did not see a dose-dependent effect of iron sucrose on complement (data not shown). However, our subgroups were small and therefore our conclusions are limited. Finally, factors such as genetic predisposition and patient characteristics, e.g., age and sex, might also impact iron-induced complement activation (17).

Clinically, complement activation can lead to acute and chronic effects. Acute complement activation by IV iron could lead to CARPA, a hypersensitivity reaction that is complement mediated (5). In the current study no hypersensitivity reactions 
A

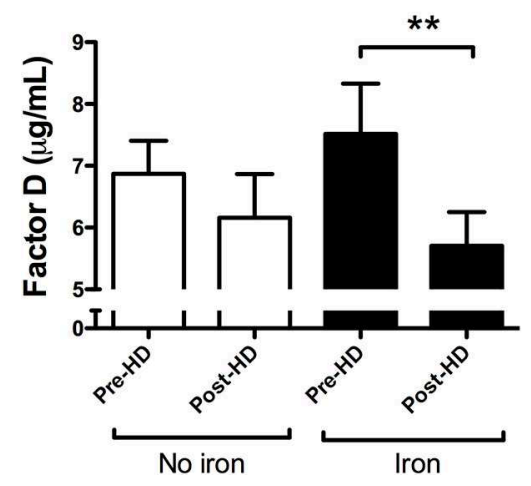

B

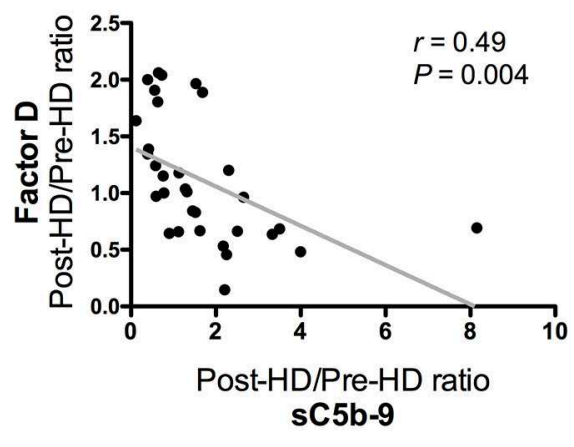

FIGURE 5 | The relative decrease in factor D correlates with the relative increase of soluble C5b-9 by intravenous iron in hemodialysis patients. (A) Plasma levels of factor $\mathrm{D}$ were determined in 32 hemodialysis (HD) patients during two consecutive dialysis sessions, one with intravenous iron (Iron), and one without (No iron). During each dialysis session, factor $\mathrm{D}$ levels were determined at baseline and after $4 \mathrm{~h}$. Data are presented as mean and SEM. (B) The correlation between the relative change of sC5b-9 and factor D between the two HD sessions. Correlations were evaluated using the Spearman rank correlation coefficient and paired sample $t$-test was used to compare values before and after iron infusion. $P<0.05$ were considered to be statistically significant $\left({ }^{\star \star} P<0.01\right)$.
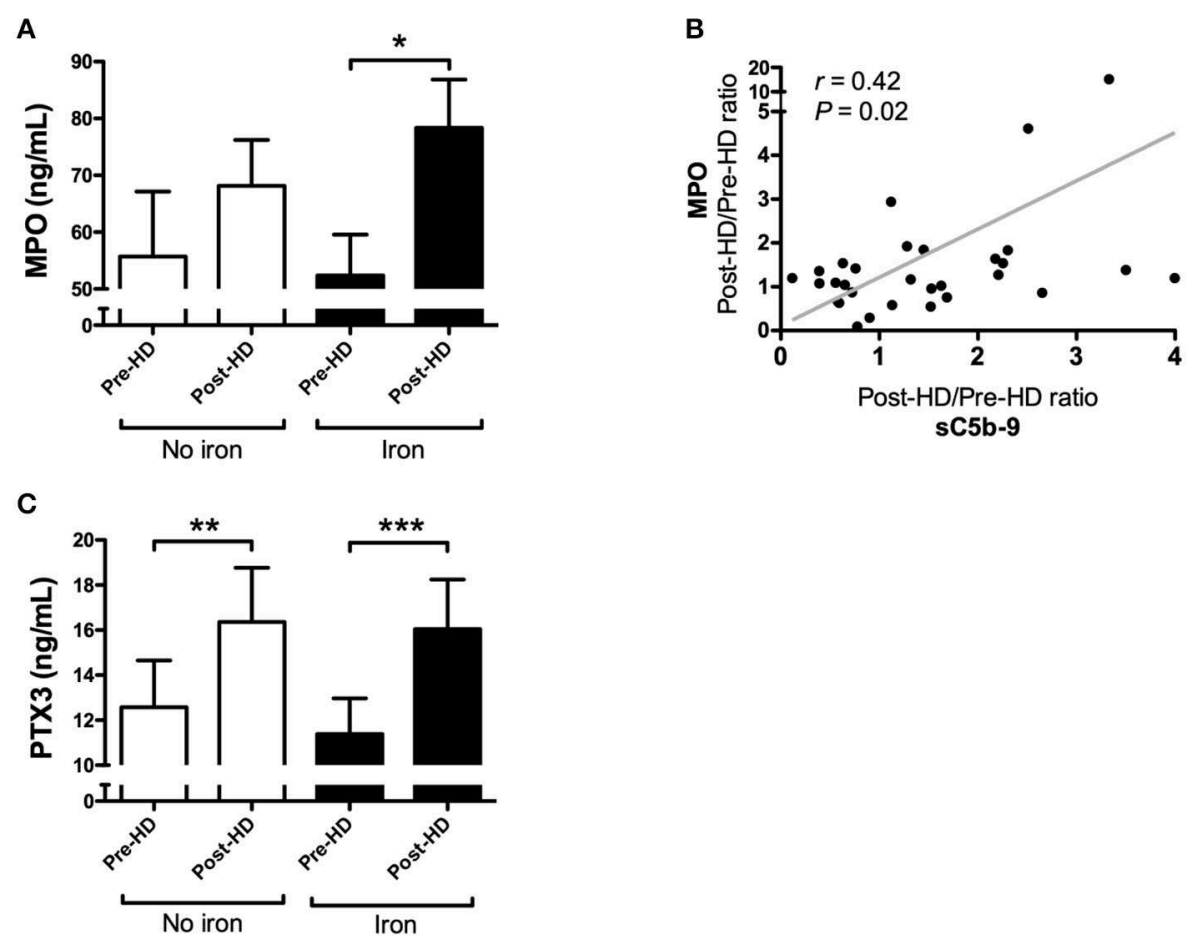

FIGURE 6 | Myeloperoxidase correlates with the relative increase of soluble C5b-9 in the dialysis session with iron, whereas pentraxin-3 increases in both dialysis sessions. (A) Plasma levels of myeloperoxidase (MPO) were determined in 32 hemodialysis (HD) patients during two consecutive dialysis sessions, one with intravenous iron (Iron), and one without (No iron). During each dialysis session, MPO levels were determined at baseline and after $4 \mathrm{~h}$. Data are presented as mean and SEM. (B) The correlation between the relative change of sC5b-9 and MPO between the two HD sessions. Correlations were evaluated using the Spearman rank correlation coefficient and paired sample $t$-test was used to compare values before and after iron infusion. $P<0.05$ were considered to be statistically significant. (C) Furthermore, plasma levels of pentraxin-3 (PTX3) were determined in 32 hemodialysis patients during two consecutive dialysis sessions, one with intravenous iron (Iron), and one without (No iron). During each dialysis session, PTX3 levels were determined at baseline and after $4 \mathrm{~h}$. Data are presented as mean and SEM. The paired sample $t$-test was used to compare values before and after iron infusion. $P$-values $<0.05$ were considered to be statistically significant $\left({ }^{\star} P<0.05\right.$, ${ }^{\star \star} P<0.01$, $\left.{ }^{\star \star \star} P<0.001\right)$

occurred, which is compatible with the fact that we did not see a 5-10-fold increase in sC5b-9, which is necessary for the development of CARPA (16). Therefore, our studies only function as a proof of principle that IV iron can induce complement activation in-vivo, but do not prove nor exclude the concept of CARPA. Moreover, chronic complement activation by 
IV Iron could contribute to prolonged/long-term oxidative stress and inflammation as well as lead to complement consumption thereby increasing infection risk. Although a recent study by Macdougall et al. demonstrated the superiority of a highdose iron-sucrose regimen compared to a low-dose regimen, limitations from their study design should be taken into account (4). First, no control group without iron administration was included, for obvious reasons. Second, regimen with other iron formulations were not compared. As such, the safety about long-term IV iron administration, particularly iron sucrose, is still moot.

MPO is a protein primarily released by activated neutrophils (18). MPO has been considered an important pathophysiological factor in oxidative stress, contributing to the activation of pro-atherogenic and inflammatory pathways, and to a higher cardiovascular risk in CKD patients $(19,20)$. In addition, MPO release has also been linked to iron infusion in an animal model (21). We therefore selected MPO as a potential marker of IV iron induced oxidative stress. Indeed, our results show that iron administration can contribute to increased levels of MPO, most likely through complement activation. We speculate that ironinduced complement activation leads to neutrophil activation resulting in the increased secretion of MPO, as shown by March et al., and this could potentially serve as a positive feedback loop for further complement activation (22-25). The significant increase in MPO levels was only seen in the context of dialysis. Possibly, the inflammatory environment caused by the dialysis procedure primed the neutrophils, subsequently making them more prone to complement activation-mediated oxidative stress, as supported by earlier studies (26-28).

Previously, Malindretos et al. studied the effect of slow infused IV iron on inflammation by measuring IL-6, CRP, and TNF- $\alpha$ (29). In their study, HD itself resulted in an increase of inflammatory parameters, while IV iron did not lead to further increase in these markers (29). Therefore, we proceeded to investigate iron-induced inflammation by using a different marker, namely PTX3 which was previously shown to be a sensitive early marker of inflammation induced by HD (3032). Although our results confirmed the increase of PTX3 levels during $\mathrm{HD}$, there was no further increase with iron administration, nor a correlation with complement activation. Possibly, the inflammatory environment of HD blurs the effect of iron on PTX3.

The strengths of our study include the use of two distinct populations (dialysis and non-dialysis) and the size of our groups. In the dialysis group, an important detail of our study design is the comparison of two different dialysis sessions in the same patient, thereby controlling for the inter-individual response to dialysis and to iron $(33,34)$. In addition, the

\section{REFERENCES}

1. Macdougall IC, Bircher AJ, Eckardt K-U, Obrador GT, Pollock CA, Stenvinkel $\mathrm{P}$, et al. Iron management in chronic kidney disease: conclusions from a "Kidney Disease: Improving Global Outcomes" (KDIGO) Controversies Conference. Kidney Int. (2016) 89:28-39. doi: 10.1016/J.KINT.2015.10.002 panel of complement measurements that were involved in the present study permitted insight in the complement pathways that leads to iron-induced complement activation. Limitations of our study include the use of only two available iron preparations. Although we compared two of the most commonly used iron formulations, not all available iron formulations were investigated. Testing low molecular weight iron dextran would be especially interesting since it gave the strongest complement activation in previous in-vitro analysis. Furthermore, we did not test different infusion rates and samples were collected only $1 \mathrm{~h}$ after IV iron. Collecting samples at other time points would give us more detailed information. Lastly, the results in the current study are associations and therefore causality cannot be proven. Unfortunately, there was no follow up and therefore we cannot evaluate long-term effects of IV iron.

In conclusion, IV iron sucrose leads to complement activation in-vivo, which partially mediates iron-induced oxidative stress. Our findings suggests that complement activation by IV iron in-vivo possibly occurs via AP, although a contribution of the LP cannot be excluded. However, the mechanisms through which iron can activate the complement system remain limited and warrant further research. Lastly, inflammation induced by IV iron is not related to PTX3 and therefore other inflammatory markers should be investigated.

\section{DATA AVAILABILITY}

All datasets generated for this study are included in the manuscript and/or the supplementary files.

\section{ETHICS STATEMENT}

The study was approved by the local ethical committee and performed according to the principles of the declaration of Helsinki. All participants gave informed consent.

\section{AUTHOR CONTRIBUTIONS}

$\mathrm{BF}, \mathrm{MG}, \mathrm{FP}, \mathrm{MD}$, and MS: research idea and study design. BF, MG, and FP: data acquisition. BF, MG, FP, CF, MP, $\mathrm{SB}, \mathrm{MD}, \mathrm{CG}$, and $\mathrm{MS}$ : data analysis and interpretation. BF, MG, and FP: statistical analysis and wrote the manuscript. All authors were involved in editing, read, and approved the final manuscript.

\section{ACKNOWLEDGMENTS}

We thank Anita Meter-Arkema and Daniela Amaro for their excellent technical assistance. 
4. Macdougall IC, White C, Anker SD, Bhandari S, Farrington K, Kalra PA, et al. Intravenous iron in patients undergoing maintenance hemodialysis. $\mathrm{N} \mathrm{Engl} \mathrm{J}$ Med. (2018). doi: 10.1056/NEJMoa1810742

5. Szebeni J. Complement activation-related pseudoallergy: a stress reaction in blood triggered by nanomedicines and biologicals. Mol Immunol. (2014) 61:163-73. doi: 10.1016/j.molimm.2014.06.038

6. Ricklin D, Reis ES, Lambris JD. Complement in disease: a defence system turning offensive. Nat Rev Nephrol. (2016) 12:383-401. doi: 10.1038/nrneph.2016.70

7. Wysowski DK, Swartz L, Vicky Borders-Hemphill B, Goulding MR, Dormitzer C. Use of parenteral iron products and serious anaphylactic-type reactions. Am J Hematol. (2010) 85:650-4. doi: 10.1002/ajh.21794

8. Hempel JCJC, Poppelaars F, Gaya Da Costa M, Franssen CFMCFM, De Vlaam TPGTPG, Daha MRMR, et al. Distinct in vitro complement activation by various intravenous iron preparations. Am J Nephrol. (2017) 45:49-59. doi: 10.1159/000451060

9. Verhoef JJF, de Groot AM, van Moorsel M, Ritsema J, Beztsinna N, Maas C, et al. Iron nanomedicines induce Toll-like receptor activation, cytokine production and complement activation. Biomaterials. (2017) 119:68-77. doi: 10.1016/j.biomaterials.2016.11.025

10. Hiemstra PS, Langeler E, Compier B, Keepers Y, Leijh PC, van den Barselaar MT, et al. Complete and partial deficiencies of complement factor D in a Dutch family. J Clin Invest. (1989) 84:1957-61. doi: 10.1172/JCI114384

11. Dobó J, Szakács D, Oroszlán G, Kortvely E, Kiss B, Boros E, et al. MASP3 is the exclusive pro-factor D activator in resting blood: the lectin and the alternative complement pathways are fundamentally linked. Sci Rep. (2016) 6:31877. doi: $10.1038 /$ srep 31877

12. Bhandari S, Pereira D, Chappell H, Drakesmith H. Intravenous irons: from basic science to clinical practice. Pharmaceuticals. (2018) 11:82. doi: $10.3390 / \mathrm{ph} 11030082$

13. Fell LH, Zawada AM, Rogacev KS, Seiler S, Fliser D, Heine GH. Distinct immunologic effects of different intravenous iron preparations on monocytes. Nephrol Dial Transplant. (2014) 29:809-22. doi: 10.1093/ndt/gft524

14. Szebeni J. Complement activation-related pseudoallergy caused by liposomes, micellar carriers of intravenous drugs, and radiocontrast agents. Crit Rev Ther Drug Carrier Syst. (2001) 18:567-606. doi: 10.1007/1-4020-8056-5_17

15. Auerbach $M$, Ballard $H$. Clinical use of intravenous iron: administration, efficacy, and safety. Hematology. (2010) 2010:338-47. doi: 10.1182/asheducation-2010.1.338

16. Chanan-Khan A, Szebeni J, Savay S, Liebes L, Rafique NM, Alving CR, et al. Complement activation following first exposure to pegylated liposomal doxorubicin (Doxil): possible role in hypersensitivity reactions. Ann Oncol. (2003) 14:1430-7. doi: 10.1093/annonc/mdg374

17. Gaya Da Costa M, Poppelaars F, van Kooten C, Mollnes TE, Tedesco F, Würzner R, et al. Age and sex-associated changes of complement activity and complement levels in a healthy Caucasian population. Front Immunol. (2018) 9:2664. doi: 10.3389/FIMMU.2018.02664

18. Kisic B, Miric D, Dragojevic I, Rasic J, Popovic L. Role of myeloperoxidase in patients with chronic kidney disease. Oxid Med Cell Longev. (2016) 2016:1069743. doi: 10.1155/2016/1069743

19. Afshinnia F, Zeng L, Byun J, Gadegbeku CA, Magnone MC, Whatling C, et al. Myeloperoxidase levels and its product 3-chlorotyrosine predict chronic kidney disease severity and associated coronary artery disease. Am J Nephrol. (2017) 46:73-81. doi: 10.1159/000477766

20. Kitabayashi C, Naruko T, Sugioka K, Yunoki K, Nakagawa M, Inaba M, et al. Positive association between plasma levels of oxidized low-density lipoprotein and myeloperoxidase after hemodialysis in patients with diabetic end-stage renal disease. Hemodial Int. (2013) 17:557-67. doi: 10.1111/hdi.12049

21. Xiao X, Saha P, Yeoh BS, Kumar MV. Myeloperoxidase deficiency attenuates dietary and systemic iron-induced toxicity. I Immunol. (2018) 200:170.8. doi: 10.1016/j.jnutbio.2018.08.003
22. March DR, Proctor LM, Stoermer MJ, Sbaglia R, Abbenante G, Reid RC, et al. Potent cyclic antagonists of the complement C5a receptor on human polymorphonuclear leukocytes. relationships between structures and activity. Mol Pharmacol. (2004) 65:868-79. doi: 10.1124/mol.65.4.868

23. O’Flynn J, Dixon KO, Faber Krol MC, Daha MR, van Kooten C. Myeloperoxidase directs properdin-mediated complement activation. J Innate Immun. (2014) 6:417-25. doi: 10.1159/000356980

24. Vogt W. Complement activation by myeloperoxidase products released from stimulated human polymorphonuclear leukocytes. Immunobiology. (1996) 195:334-46. doi: 10.1016/S0171-2985(96)80050-7

25. Camous L, Roumenina L, Bigot S, Brachemi S, Frémeaux-Bacchi V, Lesavre $\mathrm{P}$, et al. Complement alternative pathway acts as a positive feedback amplification of neutrophil activation. Blood. (2011) 117:1340-9. doi: 10.1182/blood-2010-05-283564

26. Garneata L. Intravenous iron, inflammation, and oxidative stress: is iron a friend or an enemy of uremic patients? J Ren Nutr. (2008) 18:40-5. doi: 10.1053/j.jrn.2007.10.009

27. Li X, Kshirsagar AV, Brookhart MA. Safety of intravenous iron in hemodialysis patients. Hemodial Int. (2017) 21:S93-103. doi: 10.1111/hdi.12558

28. Poppelaars F, Faria B, Gaya da Costa M, Franssen CFM, van Son WJ, Berger SP, et al. The complement system in dialysis: a forgotten story? Front Immunol. (2018) 9:71. doi: 10.3389/fimmu.2018.00071

29. Malindretos P, Sarafidis PA, Rudenco I, Raptis V, Makedou K, Makedou A, et al. Slow intravenous iron administration does not aggravate oxidative stress and inflammatory biomarkers during hemodialysis: a comparative study between iron sucrose and iron dextran. Am J Nephrol. (2007) 27:572-9. doi: 10.1159/000107928

30. Sjöberg B, Qureshi AR, Anderstam B, Alvestrand A, Bárány P. Pentraxin 3, a sensitive early marker of hemodialysis-induced inflammation. Blood Purif. (2012) 34:290-7. doi: 10.1159/000342630

31. Tong M, Carrero JJ, Qureshi AR, Anderstam B, Heimbürger O, Bárány P, et al. Plasma pentraxin 3 in patients with chronic kidney disease: associations with renal function, protein-energy wasting, cardiovascular disease, and mortality. Clin J Am Soc Nephrol. (2007) 2:889-97. doi: 10.2215/CJN.00870207

32. Jenny NS, Blumenthal RS, Kronmal RA, Rotter JI, Siscovick DS, Psaty BM. Associations of pentraxin 3 with cardiovascular disease: the MultiEthnic Study of Atherosclerosis. J Thromb Haemost. (2014) 12:999-1005. doi: $10.1111 /$ jth. 12557

33. Poppelaars F, Gaya da Costa M, Berger SP, Assa S, Meter-Arkema AH, Daha MR, et al. Strong predictive value of mannose-binding lectin levels for cardiovascular risk of hemodialysis patients. J Transl Med. (2016) 14:236. doi: 10.1186/s12967-016-0995-5

34. Poppelaars F, Gaya da Costa M, Faria B, Berger SP, Assa S, Daha MR, et al. Intradialytic complement activation precedes the development of cardiovascular events in hemodialysis patients. Front Immunol. (2018) 9:2070. doi: 10.3389/fimmu.2018.02070

Conflict of Interest Statement: CG received speaking fees and research funding from Vifor Pharma.

The remaining authors declare that the research was conducted in the absence of any commercial or financial relationships that could be construed as a potential conflict of interest.

Copyright (® 2019 Faria, Gaya da Costa, Poppelaars, Franssen, Pestana, Berger, Daha, Gaillard and Seelen. This is an open-access article distributed under the terms of the Creative Commons Attribution License (CC BY). The use, distribution or reproduction in other forums is permitted, provided the original author(s) and the copyright owner(s) are credited and that the original publication in this journal is cited, in accordance with accepted academic practice. No use, distribution or reproduction is permitted which does not comply with these terms. 